\title{
Adult female fragile $X$ premutation carriers exhibit age- and CGG repeat length-related impairments on an attentionally based enumeration task
}

\author{
Naomi J. Goodrich-Hunsaker ${ }^{1}$, Ling M. Wong' ${ }^{2}$, Yingratana McLennan², Flora Tassone ${ }^{2,3}$, Danielle Harvey , \\ Susan M. Rivera ${ }^{1,2,5}$ and Tony J. Simon ${ }^{2,6}$
}

NeuroTherapeutics Research Institute, University of California Davis Medical Center, Sacramento, CA, USA

${ }^{2}$ Medical Investigation of Neurodevelopmental Disorders Institute, University of California Davis Medical Center, Sacramento, CA, USA

${ }^{3}$ Department of Biochemistry and Molecular Medicine, University of California at Davis, Davis, CA, USA

${ }^{4}$ Department of Public Health Sciences, University of California at Davis, Davis, CA, USA

${ }^{5}$ Department of Psychology, University of California at Davis, Davis, CA, USA

${ }^{6}$ Department of Psychiatry and Behavioral Sciences, University of California at Davis, Davis, CA, USA

\section{Edited by:}

Silvia A. Bunge, University of California at Berkeley, USA

\section{Reviewed by:}

Gaia Scerif, University of Oxford, UK Daniel Ansari, The University of

Western Ontario, Canada

\section{${ }^{*}$ Correspondence:}

Naomi J. Goodrich-Hunsaker, Medical Investigation of Neurodevelopmental Disorders Institute, University of California Davis Medical Center, 2825 50th Street, Room 1362, Sacramento, CA 95817, USA.

e-mail: naomihunsaker@me.com
The high frequency of the fragile $X$ premutation in the general population and its emerging neurocognitive implications highlight the need to investigate the effects of the premutation on lifespan cognitive development. Until recently, cognitive function in fragile X premutation carriers ( $\mathrm{XXPS}$ ) was presumed to be unaffected by the mutation. Although as a group fXPCs did not differ from healthy controls (HCs), we show that young adult female fXPCs show subtle age- and significant fragile X mental retardation 1 (FMR1) gene mutation-modulated cognitive function as tested by a basic numerical enumeration task. These results indicate that older women with the premutation and fXPCs with greater CGG repeat lengths were at higher risk for difficulties in the deployment of volitional attention required to count 5-8 items, but spared performance when spatial shifts of attention were minimized to subitize a few (1-3). Results from the current study add to a growing body of evidence that suggests the premutation allele is associated with a subtle phenotype and implies that the cognitive demands necessary for counting are less effectively deployed in female fXPCs compared to HCs.

Keywords: X-linked genetic disease

\section{INTRODUCTION}

The fragile $\mathrm{X}$ mental retardation 1 (FMR1) gene is polymorphic for the length of a CGG trinucleotide repeat in the $5^{\prime}$ untranslated region located on the Xq27.3 site of the X chromosome (Verkerk et al., 1991). In the general population there are $<45$ CGG repeats in the FMR1 gene. Depending on the size of the CGG repeat expansion, the mutation can be categorized as full mutation ( $>200$ CGG repeats), which underlies fragile X syndrome (FXS), the most common inherited form of intellectual disability in males (Schneider et al., 2009). If the expansion is between 55 and 200 CGG repeats, then the individual is a fragile X premutation carrier (fXPCs, Hagerman and Hagerman, 2004; Garcia-Arocena and Hagerman, 2010). The full mutation results in silencing of the FMR1 gene and absence or limited FMR1 protein (FMRP) expression, whereas the premutation allele results in a three- to eight-fold increase in FMR1 mRNA levels in leukocytes and decreased FMRP levels due to translational inefficiency of the mutant FMR1 mRNA (Hagerman and Hagerman, 2004; Garcia-Arocena and Hagerman, 2010). Overall, it is estimated that 1 in 260-813 males and 1 in 113-259 females in the population are fXPCs (Hagerman, 2008). A major clinical consequence of the premutation allele, fragile X-associated tremor/ataxia syndrome (FXTAS), is a late-onset (>50 years old) neurodegenerative disorder that affects nearly $40 \%$ of male and $8-16 \%$ of female fXPCs (Jacquemont et al., 2004). FXTAS is associated with tremors, gait ataxia, Parkinsonism, and short-term memory and executive function impairments (Bourgeois et al., 2009).
To date, there are relatively few studies conducted on fXPCs who are asymptomatic for FXTAS (i.e., non-FXTAS), and it remains controversial whether asymptomatic, young, adult female fXPCs show cognitive impairments. Several studies report no evidence for neurocognitive implications in fXPCs younger than 50 years old (Reiss et al., 1993; Hunter et al., 2008, 2010). Other studies suggest that the premutation allele results in a subtle cognitive phenotype with reported impairments across various domains, including magnitude comparison (Goodrich-Hunsaker et al., 2011a), working memory and executive function (Loesch et al., 2003; Cornish et al., 2008), memory (Moore et al., 2004), and arithmetic (Lachiewicz et al., 2006). There is also evidence of learning differences, including deficiencies in mathematics, and attentional impairments (Hagerman et al., 1992; Steyaert et al., 1992). In some cases, these impairments appeared to be modulated by age and length of the CGG repeat expansion (Cornish et al., 2008, 2009; Goodrich-Hunsaker et al., 2011a). To add to this controversy, there is evidence that young female AXPCs may actually outperform healthy control (HC) groups in some domains. Specifically, young female AXPCs were shown to have faster reaction times compared to young $\mathrm{HC}$ adults who were not fragile X carriers (Steyaert et al., 1994; Goodrich-Hunsaker et al., 2011b).

Quantitative and numerical impairments are highly characteristic of individuals with FXS (Grigsby et al., 1990; Brainard et al., 1991; Bennetto et al., 2001; Mazzocco, 2001). Thus, it is possible 
that the premutation allele might result in a similar, but attenuated full mutation phenotype given that both the full mutation and premutation carriers of the FXS produce varying levels of reduced FMRP expression. Recent reports of poor magnitude comparison (Goodrich-Hunsaker et al., 2011a) and arithmetic abilities (Lachiewicz et al., 2006) in women who carry the premutation allele motivated the current study, the aim of which was to determine if numerical enumeration impairments existed in young adult female fXPCs, since they should be the least affected fragile X subpopulation. Unlike males, females have a second, unaffected FMR1 gene that is expressed randomly in $50 \%$ of the cells.

In the current study, we assessed performance in female fXPCs compared to female HCs on a basic numerical enumeration task. In the task, enumeration typically proceeds in one of two modes that differentially depend on spatial attention processes (Trick and Pylyshyn, 1993, 1994; Sathian et al., 1999; Piazza et al., 2002). These are referred to as subitizing and counting. Subitizing is a fast and accurate process that works only with a small set of items, usually up to 3 or 4 . There is a minimal increase in reaction time as a function of the number of items to enumerate (less than $100 \mathrm{~ms} / \mathrm{item}$ ) and error rates are also low. Conversely, counting (usually more than 4 items) requires accurate serial object detection and individuation during visual search to determine the number of items in a display, and is accomplished by shifts in spatial attention. It is therefore a much slower and more error-prone process. Reaction time increases dramatically as a function of the number of objects to enumerate (more than $250 \mathrm{~ms} /$ item). We also investigated the possibility that the older fXPCs, or those with greater genetic "dosage" as measured by larger allele size or elevated FMR1 mRNA levels, may perform more poorly in the counting mode of enumeration task.

\section{MATERIALS AND METHODS PARTICIPANTS}

Participants were 50 females aged 21-42, including $21 \mathrm{HCs}$ and 29 fXPCs. The mean age $( \pm \mathrm{SD})$ for fXPCs was $34.28 \pm 4.38$ years and for HCs was $31.72 \pm 7.06$ years. The two groups did not differ in age, $t=-1.58, p=0.12$, or Full-Scale IQ (FSIQ), $t=-1.32$, $p=0.20$ (see Table 1). Participants were recruited through the NeuroTherapeutics Research Institute (NTRI) at the Medical Investigation of Neurodevelopmental Disorders (M.I.N.D.) Institute of the University of California Davis Medical Center. This study was approved by the University of California Davis Institutional Review Board and conformed to institutional and federal guidelines for the protection of human participants. Written informed consent was obtained before behavioral testing from all participants.

\section{PSYCHOLOGICAL ASSESSMENT}

Cognitive ability was based on FSIQ using either the Wechsler Adult Intelligence Scale, third edition (Wechsler, 1997) or the Wechsler Abbreviated Scale of Intelligence (Wechsler, 1999). IQ data were available for 15/21 HCs and 22/29 fXPCs.

\section{MOLECULAR ANALYSIS}

As previously described (Tassone et al., 2008) genomic DNA was isolated from peripheral blood leucocytes using standard methods (Puregene Kit; Gentra Inc., Valencia, CA, USA). Repeat size was determined using Southern blot and PCR amplification of genomic DNA. The activation ratio (AR), indicating the percent of cells that carry the normal allele on the active X chromosome, was calculated by Southern blot. All quantifications of FMR1 mRNA were performed using a 7900 Sequence detector (PE Biosystems).

\section{ORAL MOTOR SIMPLE REACTION TIME TASK}

As previously described (Goodrich-Hunsaker et al., 2011b), participants were asked to indicate as quickly as possible, by speaking "GO" into a microphone, whenever a picture of a friendly alien appeared in the doorway of a house. The friendly alien remained on the screen until the participant responded. The task consisted of 60 consecutive trials. Delays between trials were set to one of three intervals $(400,800$, or $1200 \mathrm{~ms})$, which were presented in random order to minimize anticipatory responses. Response time was recorded as the primary dependent variable.

\section{ENUMERATION TASK}

The enumeration task was presented on a 2-GHz Intel Core 2 Duo HP Compaq dc7700 Small Form Factor PC equipped with 1 GB of RAM running SuperLab version 4.0.7b (Cedrus Corporation, San Pedro, CA, USA). Participants were asked to say into a microphone as quickly and accurately as possible the number of objects seen on the screen. To begin each trial, the participant looked at the fixation point on the computer monitor. Once the participant was ready, the stimuli were presented. Target stimuli consisted of one to eight bright green rectangles, measuring $0.25^{\circ} \times 0.24^{\circ}$ on a red background square with $2^{\circ}$ sides when viewed from a distance of $60 \mathrm{~cm}$. Example stimuli are presented in Figure 1. Targets were visible on the screen until the participant responded, at which point the vocal response terminated the trial and the timer. The experimenter, who was seated in a position from which the screen was not visible, recorded the participant's response using the computer's keyboard. Response time and error rate were recorded as the dependent variables.

Table 1 | Participant descriptive statistics and FMR1 measures.

\begin{tabular}{|c|c|c|c|c|c|c|c|c|c|c|}
\hline & \multicolumn{4}{|c|}{ Healthy control } & \multicolumn{4}{|c|}{ Fragile $\mathrm{X}$ premutation carrier } & \multirow[t]{2}{*}{$\boldsymbol{t}$} & \multirow[t]{2}{*}{$p$-value } \\
\hline & Mean & SD & Range & $n$ & Mean & SD & Range & $n$ & & \\
\hline Full-Scale IQ & 111.60 & 12.32 & $89-129$ & 15 & 116.95 & 11.99 & $101-144$ & 22 & -1.32 & 0.20 \\
\hline CGG repeat size & 30.07 & 1.22 & $28-32$ & 15 & 96.76 & 20.47 & $67-143$ & 29 & -12.54 & $<0.0001$ \\
\hline FMR1 mRNA level & 1.61 & 0.27 & $1.25-1.98$ & 8 & 2.34 & 0.47 & $1.55-3.46$ & 24 & -4.13 & $<0.0001$ \\
\hline
\end{tabular}



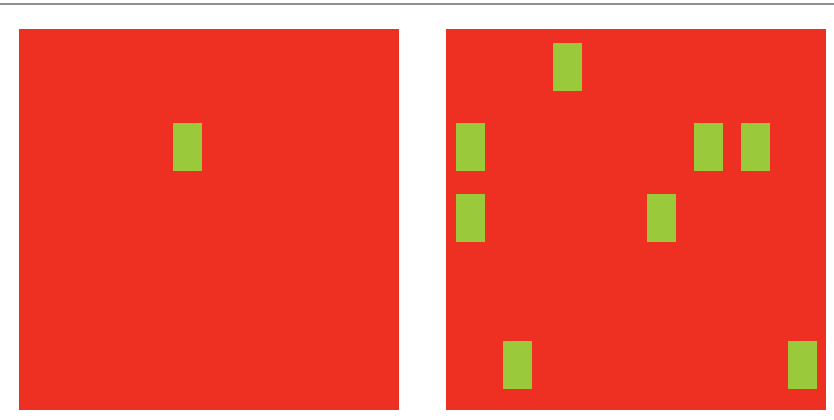

FIGURE 1 | Shown are example stimuli for the numerical enumeration task. On each trial, the participant was asked to report, by speaking into a microphone, the number of items (green rectangles). Response time and error rate were used to assess performance.

For each numerosity (1-8), there were 20 different stimuli in which the requisite number of targets was placed randomly within an invisible $4 \times 4$ grid. The experiment consisted of 5 blocks of 16 trials. All possible numerosities (1-8) were randomly distributed within a block for a total of 80 trials. A rest period was provided after every block.

\section{DATA ANALYSIS}

Data from the oral motor simple reaction time task measured basic psychomotor speed, which included the same presentation and response aspects of the enumeration task but involved minimal cognitive demands, since nothing beyond a simple oral response to a visual stimulus was required. Results of the simple reaction time were calculated as the median of reaction times across all trials and condition delays. Trials with reaction times greater than or less than three times the interquartile range or less than or equal to $150 \mathrm{~ms}$ (anticipatory responses) were excluded from the analyses.

Data from the enumeration task measured visuospatial processes as assessed by response time and error rate. These data were blocked according to the numerosity (1-8). As in our previous studies (Simon et al., 2008), anticipatory responses and outliers were excluded from the analyses. Anticipatory responses were determined to be any response time equal to or less than $150 \mathrm{~ms}$. Outliers were determined as a response time greater than or less than three times the interquartile range of the response times at each numerosity. After excluding trials with outlier responses, the median reaction time was calculated for each trial condition. GoodrichHunsaker et al. (2011b) report that oral motor reaction times were significantly faster in these same female fXPCs compared to female HC participants. In order to parse basic psychomotor speed from cognitive load, the median reaction time for each numerosity was divided by the median reaction time from the simple reaction time task. The reaction times were log-transformed to better meet the assumptions of the model.

One-way analysis of variance (ANOVA) and repeated measures ANOVA were used to assess differences between the groups on the two tasks. To estimate subitizing ranges for each group, repeated measures ANOVA models were fit to the data within a group in a sequential manner starting with 1-3 items and adding the next greater number until a quadratic trend for number of items was identified (Chi and Klahr, 1975; Lorch and Myers, 1990; Simon et al., 2008). Once the subitizing range was identified, the remaining range was identified as the counting range, and analyses focused on both ranges. Simple linear regression models with reaction times as the outcome and number of items as the independent variable were fit to each participant's data to get estimates of participant-specific slopes during the subitizing and counting ranges. These values correspond to how quickly the reaction times increased as the number of items increased. These values were then compared between the groups with a one-way ANOVA. Correlations between outcomes and age (for both groups) and molecular variables (fXPCs only) were computed within groups.

Degrees of freedom were adjusted using the Welch procedure for one-way ANOVAs when the equality of variance assumption was violated. For repeated measures ANOVAs, Greenhouse-Geisser corrections were used to correct for violations of the sphericity assumption. Assumptions for all models were checked and were met by the data. Analyses were conducted using SPSS and a $p$-value $<0.05$ was considered statistically significant.

\section{RESULTS}

\section{MOLECULAR ANALYSES}

Molecular data were available for 15/21 HC participants and 29/29 fXPCs.

Descriptive statistics of CGG repeat size, FMR1 mRNA, and ARs are reported in Table 1. Within the XXPCs, as expected, FMR1 mRNA level was positively associated with CGG repeat size, Pearson's $r=0.40, p$ (two-tailed $)=0.04$. The partial correlation between CGG and FMR1 mRNA after accounting for the AR in the fXPCs was much stronger, Pearson's $r=0.53, p$ (two-tailed $)=0.005$. Prior to examining correlations between FMR1 measures and cognitive performance, we examined potential confounding effects of age by computing Pearson correlations between each of these measures and FMR1 measures. In the female fXPCs, we found no significant correlations between CGG repeat size or FMR1 mRNA and age. The correlation matrix between CGG repeats, FMR1 mRNA, ARs, and enumeration performance are reported in Table 2.

\section{ENUMERATION TASK}

There was no difference in the error rates between the two groups, $F(1,45)=1.38, p>0.25$. The average error rate ranged from $0.00 \pm 0.00$ to $9.50 \pm 0.09 \%$ for HCs and $1.11 \pm 0.03$ to $13.70 \pm 0.18 \%$ for female fXPCs. Using a repeated measures ANOVA, we found that there was no significant difference in reaction times between the two groups, $F(1,45)=1.25, p>0.27$ (see Figure 2). Reaction times increased as the number of items increased, $F(7,315)=656.61, p<0.0001$, but did not differ between the groups, $F(7,315)=1.66, p>0.12$.

Analyses to identify the subitizing range from the counting range were performed. For the female IXPC group, a significant quadratic trend emerged with 1-4 items, indicating a subitizing range from 1 to 3 items, $F(1,27)=34.29, p<0.0001$. The same subitizing range (1-3 items) and counting range (5-8 items) was used for the two groups. Because reaction time for 4 items is at a transition point between subitizing and counting, it was not included in any slope calculations. To summarize performance in the subitizing range, slopes for the linear fit lines through the points at 1-3 items were 
Table 2 | Correlation matrix.

Neurotypical control

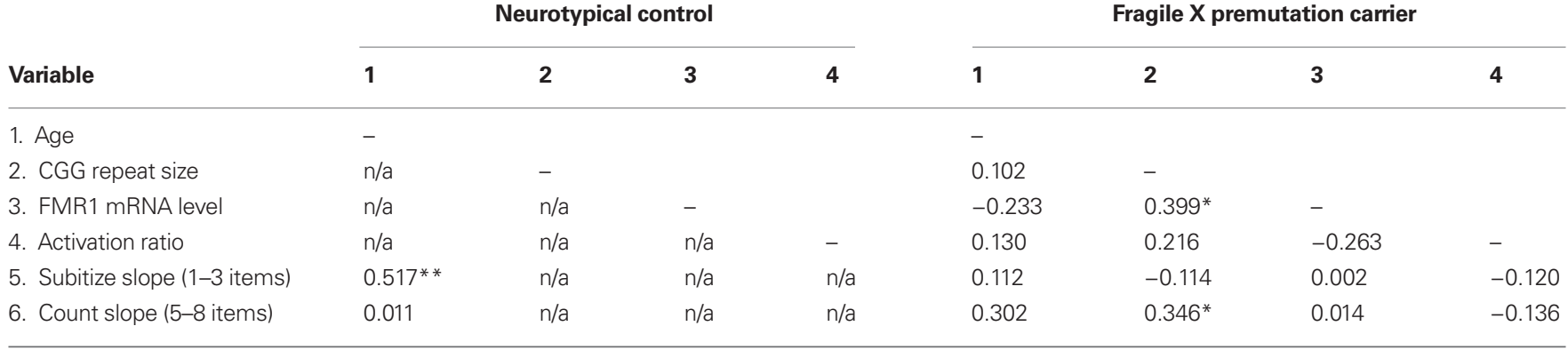

${ }^{*}$ Correlation is significant at the 0.05 level (one-tailed).

${ }^{*}$ Correlation is significant at the 0.01 level (one-tailed).
Fragile $\mathrm{X}$ premutation carrier

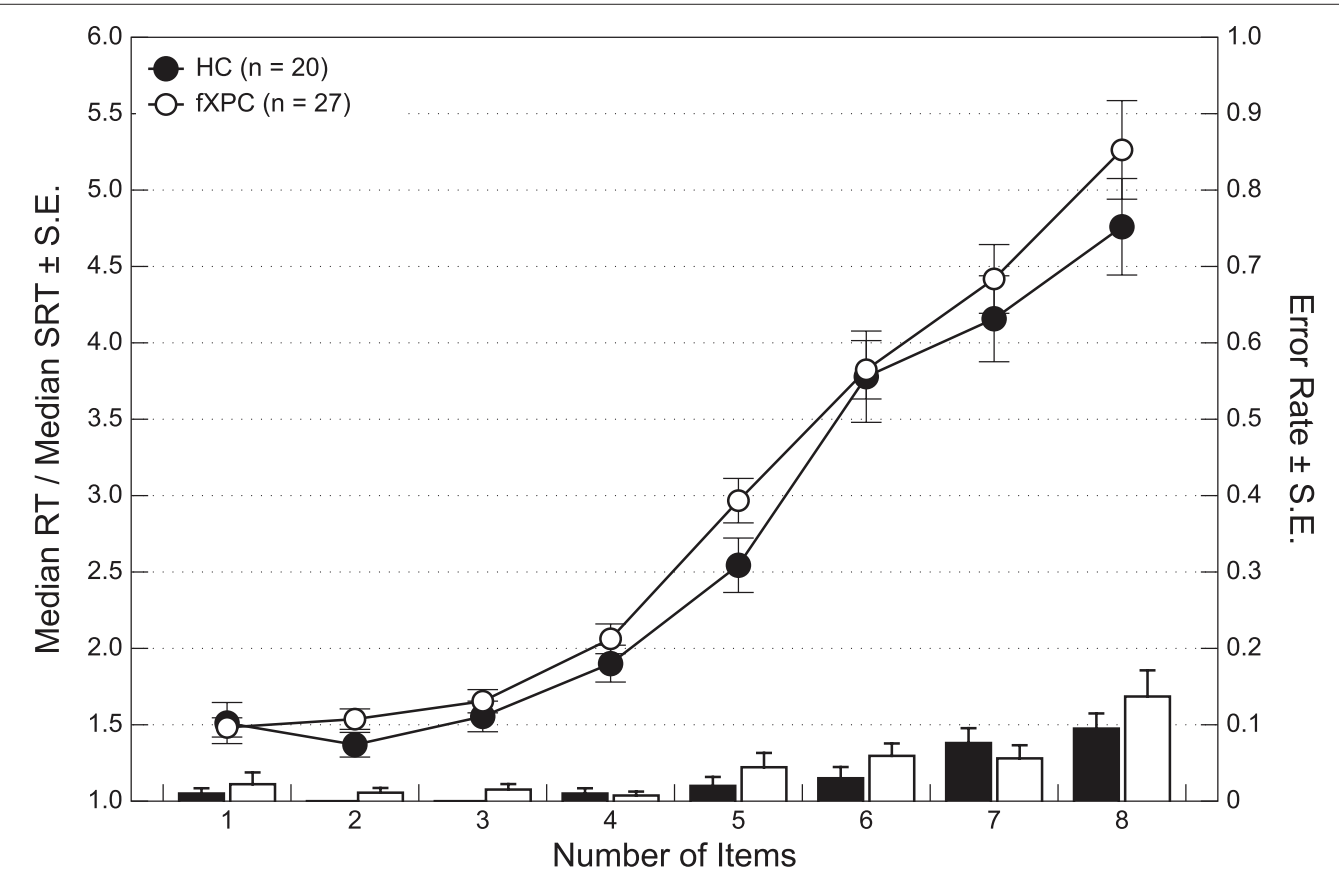

FIGURE 2 | Group analyses of response time and controlled for simple reaction time performance show that female fXPCs, as a whole, responded similarly to female HCs, $\boldsymbol{p}=\mathbf{0 . 2 7}$. Normalized response times increased as the number of items to enumerate increased $(p<0.0001)$, which did not differ between the two groups, $p=0.12$.

estimated for each participant. Similarly, for the counting range, slopes for the linear fit lines through the points at 5-8 items were estimated for each participant.

Recall that in order to parse basic psychomotor speed from cognitive load the median reaction time for each numerosity was divided by the median reaction time from the simple reaction time task, thus the slope is expressed in terms of an arbitrary unit. The average subitizing range slope was $0.08 \pm 0.20$ arbitrary unit/item for HCs and $0.09 \pm 0.12$ arbitrary unit/item for female fXPCs. The average counting range slope was $0.69 \pm 0.32$ arbitrary unit/item for HCs and $0.79 \pm 0.41$ arbitrary unit/item for female fXPCs. Slopes were not different between the groups for the subitizing range ( $p=0.85$ ) or counting range, $p=0.39$. We also calculated the slopes using raw median reaction times just to confirm that the subitizing range resulted in slopes less than $100 \mathrm{~ms} /$ item and the counting range slope was greater than $250 \mathrm{~ms} /$ item. The average subitizing range slope was $13.07 \pm 30.02 \mathrm{~ms}$ /item for HC participants and $36.66 \pm 10.39 \mathrm{~ms} /$ item for female. The average counting range slope was $354.57 \pm 35.60 \mathrm{~ms} /$ item for HCs and $348.17 \pm 34.80 \mathrm{~ms} /$ item for female fXPCs. These data match existing literature on expected subitizing and counting range slopes.

Further investigation into the slopes for the subitizing and counting range within each group assessed the association between these measures and age (both groups) and molecular variables (fXPCs only). There were two positive associations with performance in the counting range for female fXPCs. One was between age and slope [Pearson's $r=0.30, p$ (one-tailed) $=0.06$ ] and the other between CGG repeat size and slope, Pearson's $r=0.35$, 
$p($ one-tailed $)=0.03$. The degree to which reaction times increased as a function of the number of items increased (i.e., steeper slope) as the age of fXPCs increased from 20 to 42 years and as the number of their CGG trinucleotide repeats increased from 67 toward 143. We confirmed these results by also computing the partial correlation between age and slope accounting for CGG repeat length and between CGG repeat length and slope accounting for age. The partial correlation between CGG repeat size and the counting range slope after accounting for age in the fXPCs was still significant, Pearson's $r=0.33, p$ (one-tailed) $=0.04$. Interestingly, there was one significant association between the subitizing range slope and age for HC participants, which showed that the subitizing range slope steepened or was exaggerated with age, Pearson's $r=0.44, p$ (one-tailed $)=0.04$. Figure 3 presents the observed associations for each group. There was a significant difference in the association between slope and age by group for the subitizing range, $p=0.02$. We confirmed these correlations by also computing the partial correlation accounting for AR. The partial correlation between CGG repeat size and the counting range slope after accounting for the AR in the fXPCs was still significant, Pearson's $r=0.39, p$ (one-tailed $)=0.02$. No other partial correlations were significant between molecular variables and the subitizing range slope or the counting range slope. The correlation matrix between CGG repeats, FMR1 mRNA, ARs, and enumeration performance is presented in Table 2.

\section{DISCUSSION}

In the current study, we sought to quantify basic numerical enumeration performance in asymptomatic (i.e., non-FXTAS), young, adult female fXPCs. Given reports of poorer attentional and mathematical functioning in female adult premutation carriers, and the widely accepted relationship between attentional, spatial, and numerical cognition (Trick and Pylyshyn, 1993; Sathian et al., 1999; Hubbard et al., 2005; Simon et al., 2005; Ansari et al., 2007) we decided to investigate this relationship using a visuospatial enumeration task that robustly produces two modes of enumeration performance (Jevons, 1871; Chi and Klahr, 1975), which has been interpreted as differentially depending on the spatial attention system. Given previously reported age- and CGG repeat-modulated performance on a magnitude comparison task in this same group of premutation carriers (Goodrich-Hunsaker et al., 2011a), we also sought to explore the effects of age, CGG repeat length, and FMR1 mRNA levels with performance. Even after controlling for enhanced basic psychomotor speed in the female fXPC group, our results appeared at first glance to be consistent with those of many previous studies that show female fXPCs to be cognitively unaffected. As a group, the female fXPCs produced similar subitizing and counting responses to the HCs. However, our results indicate that female fXPCs appear to have changes in cognitive function as measured by a simple numerical enumeration task that is related to both age and size of the CGG repeat expansion.

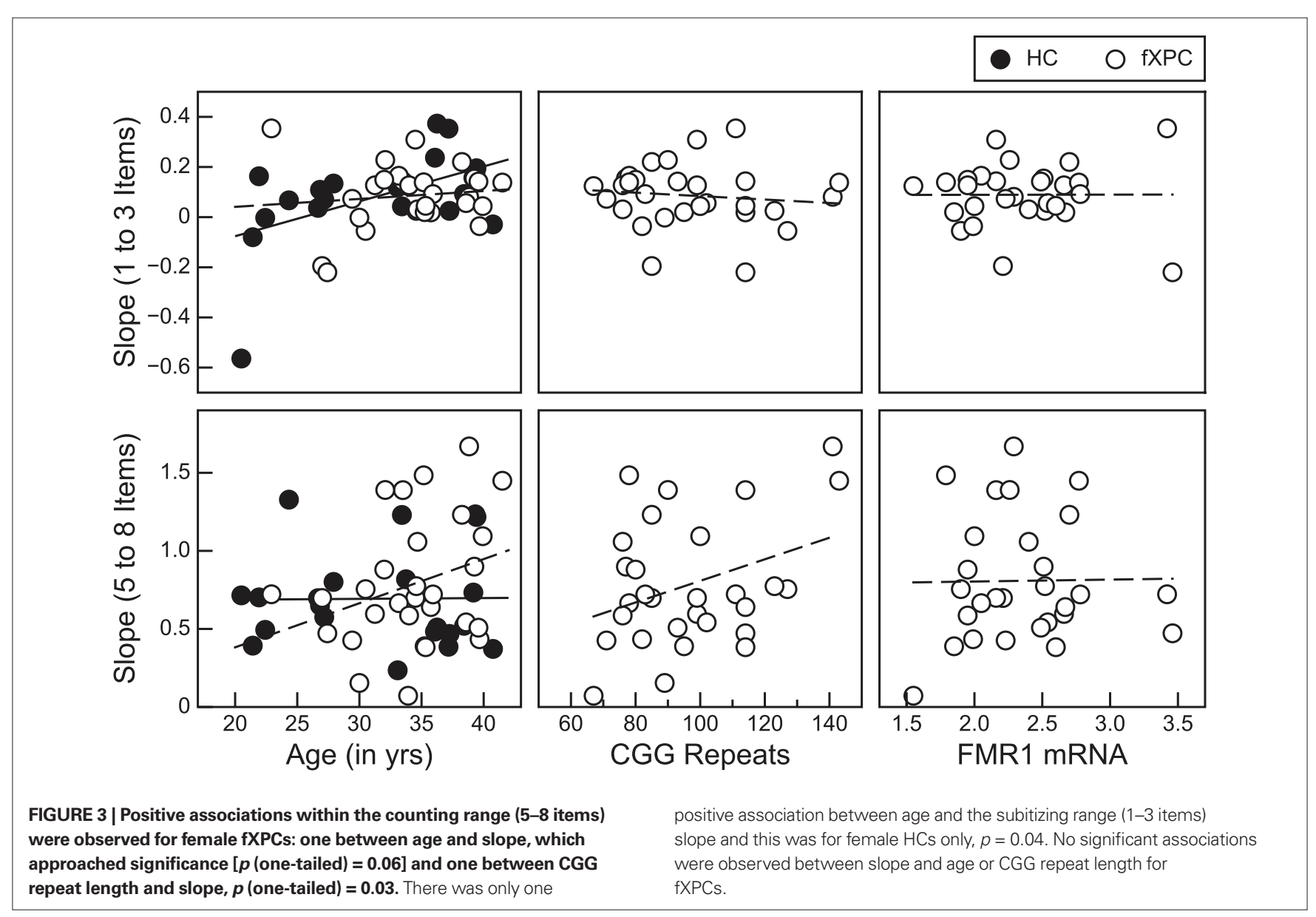


In the fXPC, but not the HC group, we found that the increase in reaction time to count 5-8 items (counting range) became more exaggerated with age, from 20 to 42 years old. This relationship was almost strong enough to reach significance at the alpha level of $p<0.05$. There was no such relationship in the fXPC group within the subitizing range ( $1-3$ items). These results indicate impairment in what is widely assumed to require the serial spatial deployment of volitional attention in order to count 5-8 items, but typical performance when spatial shifts of attention were minimized by the requirement to subitize, a few items (1-3 items). In contrast, $\mathrm{HCs}$ showed a significant increase in reaction time to subitize 1-3 items as the age of the participant increased. It remains unclear why there is a significant difference in this relationship between the XXPC and HC group and whether this implicates enhanced or impaired performance in fXPCs compared to HC participants. Inspection of Figure 3 shows little difference in terms of the spread of the slope values in the older women in either group for 1-3 items, indicating that advancing age did not introduce more impaired performance in the HC group. Instead, it appears that there are more young women in the HC group than in the IXPC group who produced rather small to negative slope values on the task. A different pattern emerges for counting 5-8 items; the spread of the slope values in the young women in either group is tight and seemingly similar, whereas in the older fXPCs there appears to be more dispersion of slope values compared to the HC participants. These results suggest that increasing age within fXPCs may be accompanied by impairments in the counting range where serial, controlled shifts of attention are generally thought to be required. These findings do not necessarily indicate any evidence of neurocognitive degeneration in the group of women carrying the fragile $\mathrm{X}$ premutation, only that age is an important factor to consider when assessing modulators of cognitive performance in fXPCs. However, these data do imply that the cognitive demands necessary for counting are less effectively deployed in older female fXPCs compared to younger fXPCs.

An even stronger relationship was found in the IXPC group when we investigated how slope values varied with CGG repeat length to count 5-8 items. There was a significant positive relationship, indicating that performance was significantly poorer as the number of CGG trinucleotide repeats increased from 67 toward 143. There was no significant relationship between CGG repeat length and slope values to subitize 1-3 items. We hypothesize that the reduction in FMRP that is assumed to increase as CGG length increases may contribute to the subtle impairments in attention necessary for counting. Diminished or absent production of FMRP in participants with the full mutation (>200 CGG repeats), which represents the biological basis for FXS, results in severe intellectual disability (Schneider et al., 2009).

Interestingly, the effect was not evident when performance was related to leukocyte FMR1 mRNA levels. This last observation is not too surprising given that leukocyte FMR1 mRNA does not reflect levels of FMR1 mRNA in the brain. The premutation allele results in a three- to eight-fold increase in leukocyte FMR1 mRNA levels and only a one- to two-fold increase in brain FMR1
mRNA levels (Tassone et al., 2000). Brain levels of FMR1 mRNA also vary widely across different brain regions (Jacquemont et al., 2004).

Clearly, some limitations exist in these very early investigations of the developmental neurocognitive endophenotype of young adults carrying the fragile $\mathrm{X}$ premutation allele. First of all, our study was cross-sectional in nature and so it should be stressed that our data do not allow us to determine whether the age effect is a significant characteristic of the fragile $\mathrm{X}$ premutation phenotype in females or if it was just representative of the sample that we tested. Further studies, especially longitudinal studies, might aid in identifying whether impairments develop at some critical point in response to environmental factors, or if they develop along a continuous trajectory of development that is increasingly divergent from HCs. Future directions will consist of testing a greater number of participants within the current age range of 20-40 years old as well as testing older participants.

Further, although performance was positively associated with CGG repeat length in the fXPC group even after controlling for ARs, we recognize that this may not be the most reliable marker of molecular pathology. However, the results of the current study are supported by previous studies showing similar relationships (Cornish et al., 2009; Goodrich-Hunsaker et al., 2011a). Without FMRP levels, the outcomes of elevated FMR1 mRNA as a result of the premutation allele are unknown. FMRP was not quantified in the participants, so no direct correlation can be drawn between markers of molecular pathology and enumeration performance.

As with previous studies, our hypothesis was that asymptomatic (i.e., non-FXTAS) adult female fXPCs might not be grossly cognitively affected compared to female HCs. There remained the possibility that subtler cognitive dysfunction may exist. We assessed whether such subtle impairments existed, and whether they were affected by age and genetic dosage. Female fXPCs did not show age- or CGG repeat length-modulated performance in the subitizing range, when spatial shifts of attention are widely thought to be minimally required in order to accomplish object detection and individuation. However, the serial deployment of attention to count 5-8 items was less efficient in the older fXPCs and in those with greater genetic "dosage" as measured by larger allele size. The results of the current study add to an expanding body of evidence that the premutation allele is associated with a subtle phenotype, the larger clinical implications of which remain currently unclear.

\section{ACKNOWLEDGMENTS}

This work was supported by National Institute of Health (NIH) grants: NIA RL1 AG032119, NINDS RL1 NS062412, and NIDA TL1 DA024854. This work was also made possible by a Roadmap Initiative grant (UL1 DE019583) from the National Institute of Dental and Craniofacial Research (NIDCR) in support of the NeuroTherapeutics Research Institute (NTRI) consortium. The $\mathrm{NIH}$ had no further role in study design; in the collection, analysis, and interpretation of data; in the writing of the report; and in the decision to submit the paper for publication. We thank the participants who made this work possible and Siddharth Srivastava for critical review and comments. 


\section{REFERENCES}

Ansari, D., Lyons, I. M., Van Eimeren, L., and $\mathrm{Xu}, \mathrm{F}$. (2007). Linking visual attention and number processing in the brain: the role of the temporoparietal junction in small and large symbolic and nonsymbolic number comparison. J. Cogn. Neurosci. 19, 1845-1853.

Bennetto, L., Pennington, B. F., Porter, D., Taylor, A. K., and Hagerman, R. J. (2001). Profile of cognitive functioning in women with the fragile X mutation. Neuropsychology 15, 290-299.

Bourgeois, J. A., Coffey, S. M., Rivera, S. M., Hessl, D., Gane, L. W., Tassone, F., Greco, C., Finucane, B., Nelson, L., Berry-Kravis, E., Grigsby, J., Hagerman, P. J., and Hagerman, R. J. (2009). A review of fragile $X$ premutation disorders: expanding the psychiatric perspective. J. Clin. Psychiatry $70,852-862$.

Brainard, S. S., Schreiner, R. A., and Hagerman, R. J. (1991). Cognitive profiles of the carrier fragile $\mathrm{X}$ woman. Am. J. Med. Genet. 38, 505-508.

Chi, M. T., and Klahr, D. (1975). Span and rate of apprehension in children and adults. J. Exp. Child Psychol. 19, 434-439.

Cornish, K. M., Kogan, C. S., Li, L., Turk, J., Jacquemont, S., and Hagerman, R. J. (2009). Lifespan changes in working memory in fragile $\mathrm{X}$ premutation males. Brain Cogn. 69, 551-558.

Cornish, K. M., Li, L., Kogan, C. S., Jacquemont, S., Turk, J., Dalton, A., Hagerman, R. J., and Hagerman, P. J. (2008). Age-dependent cognitive changes in carriers of the fragile $\mathrm{X}$ syndrome. Cortex 44, 628-636.

Garcia-Arocena, D., and Hagerman, P. J. (2010). Advances in understanding the molecular basis of FXTAS. Hum. Mol. Genet. 19, R83-R89.

Goodrich-Hunsaker, N. J., Wong, L. M., Mclennan, Y., Srivastava, S., Tassone, F., Harvey, D., Rivera, S. M., and Simon, T. J. (2011a). Young adult female fragile $\mathrm{X}$ premutation carriers show age- and genetically-modulated cognitive impairments. Brain Cogn. $75,255-260$.

Goodrich-Hunsaker, N. J., Wong, L. M., Mclennan, Y., Tassone, F., Harvey, D., Rivera, S. M., and Simon, T. J. (2011b). Enhanced manual and oral motor reaction time in young adult female fragile X premutation carriers. J. Int. Neuropsychol. Soc. 17, 746-750.

Grigsby, J. P., Kemper, M. B., Hagerman, R. J., and Myers, C. S. (1990). Neuropsychological dysfunction among affected heterozygous fragile $\mathrm{X}$ females. Am. J. Med. Genet. 35, 28-35.

Hagerman, P. J. (2008). The fragile X prevalence paradox. J. Med. Genet. $45,498-499$.

Hagerman, P. J., and Hagerman, R. J. (2004). Fragile X-associated tremor/ ataxia syndrome (FXTAS). Ment. Retard.Dev. Disabil. Res. Rev. 10,25-30.

Hagerman, R. J., Jackson, C., Amiri, K. Silverman, A. C., O'Connor, R., and Sobesky, W. (1992). Girls with fragile $\mathrm{X}$ syndrome: physical and neurocognitive status and outcome. Pediatrics $89,395-400$

Hubbard, E. M., Piazza, M., Pinel, P., and Dehaene, S. (2005). Interactions between number and space in parietal cortex. Nat. Rev. Neurosci. 6, 435-448.

Hunter, J. E., Allen, E. G., Abramowitz, A., Rusin, M., Leslie, M., Novak, G., Hamilton, D., Shubeck, L., Charen, K., and Sherman, S. L. (2008). No evidence for a difference in neuropsychological profile among carriers and noncarriers of the FMR1 premutation in adults under the age of 50. Am. J. Hum. Genet. 83, 692-702.

Hunter, J. E., Rohr, J. K., and Sherman, S. L. (2010). Co-occurring diagnoses among FMR1 premutation allele carriers. Clin. Genet. 77, 374-381.

Jacquemont, S., Hagerman, R. J., Leehey, M. A., Hall, D. A., Levine, R. A., Brunberg, J. A., Zhang, L., Jardini, T., Gane, L. W., Harris, S. W., Herman, K., Grigsby, J., Greco, C. M., BerryKravis, E., Tassone, F., and Hagerman, P. J. (2004). Penetrance of the fragile $\mathrm{X}$-associated tremor/ataxia syndrome in a premutation carrier population. JAMA 291, 460-469.

Jevons, W.S. (1871). The power of numerical discrimination. Nature 3, 281-282.

Lachiewicz, A. M., Dawson, D. V. Spiridigliozzi, G. A., and McconkieRosell, A. (2006). Arithmetic difficulties in females with the fragile $\mathrm{X}$ premutation. Am. J. Med. Genet. A $140,665-672$.

Loesch, D. Z., Bui, Q. M., Grigsby, J., Butler, E., Epstein, J., Huggins, R. M., Taylor, A. K., and Hagerman, R. J. (2003). Effect of the fragile X status categories and the fragile $\mathrm{X}$ mental retardation protein levels on executive functioning in males and females with fragile X. Neuropsychology 17, 646-657.

Lorch, R. F. Jr., and Myers, J. L. (1990). Regression analyses of repeated measures data in cognitive research. J. Exp. Psychol.Learn.Mem. Cogn. 16, 149-157.

Mazzocco, M. M. (2001). Math learning disability and math $\mathrm{LD}$ subtypes: evidence from studies of Turner syndrome, fragile $\mathrm{X}$ syndrome, and neurofibromatosis type 1. J. Learn Disabil. $34,520-533$.

Moore, C. J., Daly, E. M., Schmitz, N., Tassone, F., Tysoe, C., Hagerman, R. J. Hagerman, P.J., Morris, R. G., Murphy, K. C., and Murphy, D. G. (2004). A neuropsychological investigation of male premutation carriers of fragile X syndrome. Neuropsychologia 42 , 1934-1947.

Piazza, M., Mechelli, A., Butterworth, B., and Price, C. J. (2002). Are subitizing and counting implemented as separate or functionally overlapping processes? Neuroimage 15, 435-446.

Reiss, A. L., Freund, L., Abrams, M. T. Boehm, C., and Kazazian, H. (1993). Neurobehavioral effects of the fragile $\mathrm{X}$ premutation in adult women: controlled study. Am. J. Hum. Genet. $52,884-894$.

Sathian, K., Simon, T. J., Peterson, S., Patel, G. A., Hoffman, J. M., and Grafton, S. T. (1999). Neural evidence linking visual object enumeration and attention. J. Cogn. Neurosci. 11, 36-51.

Schneider, A., Hagerman, R. J., and Hessl, D. (2009). Fragile X syndrome - from genes to cognition. Dev. Disabil. Res. Rev. 15, 333-342.

Simon, T. J., Bearden, C. E., McDonald Mc-Ginn, D. M., and Zackai, E. (2005). Visuospatial and numerical cognitive deficits in children with chromosome 22q11.2 deletion syndrome. Cortex 41 , 145-155.

Simon, T. J., Takarae, Y., DeBoer, T., McDonald-McGinn, D. M., Zackai, E. H., and Ross, J. L. (2008). Overlapping numerical cognition impairments in children with chromosome 22q11.2 deletion or Turner syndromes. Neuropsychologia 46, 82-94.

Steyaert, J., Borghgraef, M., and Fryns, J. P. (1994). Apparently enhanced visual information processing in female fragile $\mathrm{X}$ carriers: preliminary findings. Am. J. Med. Genet. 51, 374-377.

Steyaert, J., Borghgraef, M., Gaulthier C., Fryns, J. P., and Van Den Berghe, H. (1992). Cognitive profile in adult, normal intelligent female fragile X carriers. Am. J. Med. Genet. 43 , 116-119.

Tassone, F., Hagerman, R. J., Taylor, A. K. Mills, J. B., Harris, S. W., Gane, L. W. and Hagerman, P. J. (2000). Clinical involvement and protein expression in individuals with the FMR1 premutation. Am. J. Med. Genet. 91, 144-152.

Tassone, F., Pan, R., Amiri, K., Taylor, A. K., and Hagerman, P. J. (2008). A rapid polymerase chain reaction-based screening method for identification of all expanded alleles of the fragile $X$ (FMR1) gene in newborn and highrisk populations. J. Mol. Diagn. 10, 43-49.

Trick, L. M., and Pylyshyn, Z. W. (1993). What enumeration studies can tell us about spatial attention. Evidence for limited capacity preattentive processing. J. Exp. Psychol. Hum. Percept. Perform. 19, 331-351.

Trick, L. M., and Pylyshyn, Z. W. (1994). Why are small and large numbers enumerated differently? A limitedcapacity preattentive stage in vision. Psychol. Rev. 101, 80-102.

Verkerk, A. J. M. H., Pieretti, M., Sutcliffe, J. S., Fu, Y.-H., Kuhl, D. P. A., Pizzuti, A., Reiner, O., Richards, S., Victoria, M. F., Zhang, F., Eussen, B. E., van Ommen, G.-J. B., Blonden, L. A. J., Riggins, G. J., Chastain, J. L., Kunst, C. B., Galjaard, H., Caskey, C. T., Nelson, D. L., Oostraa, B. A., and Warren, S. T. (1991). Identification of a gene (FMR-1) containing a CGG repeat coincident with a breakpoint cluster region exhibiting length variation in fragile X syndrome. Cell 65, 905-914.

Wechsler, D. (1997). WAIS-III: Wechsler Adult Intelligence Scale. San Antonio, TX: Psychological Corporation.

Wechsler, D. (1999). Wechsler Abbreviated Scale of Intelligence. San Antonio, TX: Psychological Corporation.

Conflict of Interest Statement: The authors declare that the research was conducted in the absence of any commercial or financial relationships that could be construed as a potential conflict of interest.

Received: 25 March 2011; accepted: 27 June 2011; published online: 14 July 2011. Citation: Goodrich-Hunsaker NJ, Wong LM, McLennan Y, Tassone F, Harvey D, Rivera SM and Simon TJ (2011) Adult female fragile $X$ premutation carrier exhibitage-and CGG repeat length-related impairments on an attentionally based enumeration task. Front. Hum. Neurosci. 5:63. doi: 10.3389/fnhum.2011.00063

Copyright @ 2011 Goodrich-Hunsaker, Wong, McLennan, Tassone, Harvey, Rivera and Simon. This is an open-access article subject to a non-exclusive license between the authors and Frontiers Media $S A$, which permits use, distribution and reproduction in other forums, provided the original authors and source are credited and other Frontiers conditions are complied with 